\title{
Alpha-2 Adrenergic Regulation of Melatonin Release in Chick Pineal Cell Cultures
}

\author{
Barbara L. Pratt and Joseph S. Takahashi \\ Department of Neurobiology and Physiology, Northwestern University, Evanston, Illinois 60201
}

The chick pineal gland expresses a circadian rhythm of melatonin biosynthesis, with elevated levels at night and low levels during the day. The rhythm of melatonin is regulated both by circadian oscillators located within the gland itself and by adrenergic input from the sympathetic nervous system. Previous work has shown that norepinephrine administration inhibits melatonin biosynthesis, as measured by the activity of the enzyme serotonin $\mathrm{N}$-acetyltransferase. As a first step toward understanding the mechanisms by which norepinephrine regulates melatonin production in the chick pineal, we have identified the adrenergic receptor involved. Dissociated chick pineal cell cultures were prepared and melatonin release was measured on days 5 and 6 of culture using radioimmunoassay. The effects of adrenergic agonists and antagonists on the nocturnal increase of melatonin release during the $12 \mathrm{hr}$ dark portion of a LD 12:12 light cycle were determined.

Norepinephrine inhibited melatonin release in a dose-dependent manner, with an average $E_{50}$ of $19.7 \mathrm{nM} \pm 2.23$ (SEM). Melatonin release values ranged from 100 to $4 \%$ of the level seen in control cultures, depending on the dose of norepinephrine. The physiological response to epinephrine, norepinephrine, and isoproterenol was stereospecific. The (-) stereoisomer was 6, 8, and 37 times more potent than the $(+)$ stereoisomer, respectively. $E_{50}$ values (in $n M$ ) for adrenergic agonists were as follows: alpha-methyl-(-)norepinephrine, 2.46; tramazoline, 3.06; guanabenz, 3.31; clonidine, 3.70; oxymetazoline, 4.29; (-)-epinephrine, 7.44; (-)-norepinephrine, 19.7; (-)-isoproterenol, 463; and (-)phenylephrine, 659.

Schild analysis was used to determine the relative potency of adrenergic antagonists. $\mathbf{p A}_{2}$ values for adrenergic antagonists were as follows: rauwolscine, 9.55; RX781094, 8.32; yohimbine, 8.14; phentolamine, 7.11; prazosin, 5.93; and $(-)$-propranolol, $<6$. The relative potencies of both adrenergic agonists and antagonists demonstrate that alpha-2 receptors mediate norepinephrine-induced inhibition of melatonin release in chick pineal cell cultures. The identification of alpha-2 receptors in chick pineal cells should aid in our understanding of the biochemical events initiated by receptor activation that regulate melatonin synthesis.

\footnotetext{
Received Dec. 9, 1986; revised May 8, 1987; accepted May 11, 1987.

This work was supported by NIMH Grant MH-39592, NSF Presidential Young Investigator Award DCB-8451642, and Searle Scholars Award 85-H-107 to J.S.T., and T32 HD- 07068 to B.L.P. We thank Dr. G. D. Niswender for providing the melatonin antibody.

Correspondence should be addressed to Dr. Barbara Pratt, Department of Neurobiology and Physiology, Northwestern University, Hogan Hall, Evanston, IL 60201

Copyright (C) 1987 Society for Neuroscience $0270-6474 / 87 / 113665-10 \$ 02.00 / 0$
}

The vertebrate pineal gland rhythmically produces a hormone, melatonin, that appears to subserve a diversity of physiological processes that are regulated by the day-night cycle. These include photoperiodic regulation of seasonal reproduction in mammals (Goldman, 1983), control of circadian rhythms in birds and reptiles (Gwinner and Benzinger, 1978; Underwood and Harless, 1985), regulation of retinal photoreceptor metabolism (Besharse et al., 1984), and hormonal control of melanocytes (Bagnara and Hadley, 1970). Without exception, melatonin synthesis and/or release is high during the night and low during the day. Despite the common waveform of the rhythm, the mechanisms by which melatonin is regulated vary by species.

Regulation of melatonin synthesis is well understood in the rat. Ihere is a circadian rhythm in the activity of the ratelimiting enzyme in the synthesis of melatonin, arylalkylamine $N$-acctyltransforasc (NAT; EC 2.3.1.87), as well as in the level of melatonin itself (Zatz et al., 1978; Klein et al., 1981). Both of these circadian rhythms are driven by sympathetic stimulation of the pineal gland arising from the superior cervical ganglia, which, in turn, receive input from the suprachiasmatic nuclei, which are thought to contain a circadian clock. Lesioning the suprachiasmatic nuclei or interrupting the neural pathway to the gland abolishes all pineal rhythmicity (Klein and Moore, 1979). Since the mammalian pineal gland is not photoreceptive, both circadian rhythms and the effects of environmental lighting are imposed upon the pineal through variations in the release of norepinephrine from sympathetic terminals. Norepinephrine turnover, release, and/or metabolism are highest during the night, which accounts for the increased levels of NAT and melatonin (Brownstein and Axelrod, 1974). Norepinephrine treatment in organ culture leads to an increase of melatonin synthesis (Zatz et al., 1978; Klein et al., 1981), as does electrical stimulation of the sympathetic nerves innervating the gland (Bowers and Zigmond, 1982).

The physiological organization of the avian pineal is considerably different from that of the mammalian pineal. First, the avian pineal gland contains circadian oscillators that regulate rhythmic melatonin biosynthesis (Deguchi, 1979a; Kasal et al., 1979; Takahashi et al., 1980). Denervating the gland does nut abolish circadian rhythms of indole metabolism (Ralph et al., 1975). Second, the pineal of the chick is photoreceptive when isolated in vitro (Binkley et al., 1978; Deguchi, 1979c, 1981; Wainwright and Wainwright, 1980; Hamm et al., 1983). Furthermore, circadian rhythms of melatonin release from dissociated pineal cells can be entrained by light in vitro (Robertson and Takahashi, 1987). Hence, it appears that the isolated avian pineal contains all of the components of a circadian system: a pacemaker or oscillator, a photoreceptive input, and an overt melatonin rhythm (Takahashi and Menaker, 1984a). 
Despite the capacity of the avian pineal gland for endogenous regulation of the rhythm of melatonin release, sympathetic innervation does play a role in the regulation of the melatonin rhythm. The innervation of the avian pineal gland is similar to that seen in other vertebrates (Ariëns Kappers, 1965). Norepinephrine-containing postganglionic fibers, arising from the superior cervical ganglia, provide the major innervation to the gland (Sato and Wakc, 1983). Trcatment with norepinephrine or its agonists in organ-cultured chick pineals leads to a decrease in the activity of NAT (Deguchi, 1979b), and treatment with norepinephrine in vivo inhibits serum melatonin levels in ganglionectomized birds (Cassone and Menaker, 1983). Because norepinephrine inhibits melatonin synthesis, one would expect that norepinephrine turnover, release, and/or metabolism would be highest in the daytime. This temporal relationship appears to hold (Cassone et al., 1986). The rhythm of norepinephrine turnover in the chick is elevated during the day and low at night, and is $180^{\circ}$ out of phase with the rhythm of norepinephrine turnover in the rat. Because the effect of norepinephrine is opposite in rats and chicks, the net effect in both cases is high melatonin synthesis at night and low synthesis during the day.

A complete understanding of the mechanisms regulating the daily rhythm of melatonin production will depend on an understanding of the nature of the adrenergic receptors involved. The adrenergic control of melatonin synthesis is not as well understood in the chick as it is in the rat. In the rat, we know that postsynaptic beta-adrenergic receptors stimulate the activity of NAT via receptor-modulated changes in enzyme activities and cyclic nucleotide levels (Zatz et al., 1978). Recent evidence indicates that the classic beta-adrenergic effect in the rat is potentiated by postsynaptic alpha- 1 adrenoceptors (Auerbach et al., 1981; Sugden and Klein, 1984; Sugden et al., 1984). Combined treatment of pineal glands by both alpha- 1 and betaadrenergic agonists produced a higher elevation of NAT and cAMP than did beta-adrenergic agonists administered alone. Alpha-1 agonists, by themselves, had a negligible effect. In contrast, stimulation of postsynaptic alpha-1 receptors in the sheep is primarily responsible for the nocturnal rise of melatonin (Sugden et al., 1985b). In the chick, preliminary evidence suggests that alpha adrenoceptors mediate the catecholamine-induced suppression of NAT (Deguchi, 1979b). However, these studies did not characterize the dose dependence, the location, or the subtype of the alpha receptor. Deguchi's preliminary work on the nature of the adrenergic receptor in the chick pineal was performed with short-term organ culture in which synaptic terminals still may have been present.

In order to identify the adrenergic receptors in the avian pincal gland, we have developed a dispersed cell culture system in which only postjunctional receptors are present. In this system, it is possible to analyze the effects of adrenergic agonists and antagonists in order to identify definitively the adrenergic receptor subtype responsible for the regulation of the melatonin rhythm by norepinephrine.

\section{Materials and Methods}

Chemicals. Both (+) and $(-)$ isomers of norepinephrine, epinephrine and isoproterenol $d$-bitartrate, were donated by the Sterling-Winthrop Research Institute (Rensselear, NY); guanahenz acetate by Wyeth Lahs (Philadelphia, PA); phentolamine $\mathrm{HCl}$ by CIBA Pharmaceutical Co. (Summit, NJ); (-)-propranolol $\mathrm{HCl}$ by Ayerst Labs (New York, NY); prazosin $\mathrm{HCl}$ by Pfizer Labs (New York, NY); SCH23390 and oxymetazoline by Schering (Bloomfield, $\mathrm{NJ}$ ); tramazoline $\mathrm{HCl}$ and cloni- dine $\mathrm{HCl}$ by Boehringer Ingelheim (Ridgefield, CT); S-sulpiride by Ravizza Pharmaceutical Co. (Milan, Italy); and idazoxan (RX781094) by Reckitt and Colman (Overseas) (Kingston-upon-Hull, England).

Rauwolscine $\mathrm{HCl}$ was commercially obtained from the Accurate Chemical and Scientific Co. (Westbury, NY); WB4101 from Research Biochemicals (Wayland, MA); and alpha-methyl-(-)-norepinephrine, yohimbine $\mathrm{HCl},(-)$-phenylephrine $\mathrm{HCl}$, dopamine $\mathrm{HCl},(-)$-norepinephrine bitartrate, and sodium ascorbate from the Sigma Chemical Co. (St. Louis, MO).

The following chemicals were used in the radioimmunoassay for melatonin: rabbit gamma globulin purchased from Cooper Biomedical (Malvern, PA); ${ }^{125}$ I melatonin analog purchased from Meloy Labs (Springfield, VA); and rabbit anti-melatonin (R1055 pool) antibody, generously provided by Dr. Gordon D. Niswender.

Radioimmunoassay of melatonin. Appropriate volumes $(2-6 \mu \mathrm{l})$ of each sample were assayed for melatonin content by radioimmunoassay using a modification of the method of Rollag and Niswender (1976) as described by Takahashi et al. (1980). The assay has been validated for pineal culture medium without extraction of the samples (Takahashi et al., 1980). No interference from the culture medium nor from any of the experimental drugs was detectable at the sample volumes assayed. The upper and lower limits of the assay were approximately $500 \mathrm{pg} /$ tube and $1 \mathrm{pg} /$ tube, respectively. Fifty percent inhibition was typically produced with $18 \mathrm{pg} /$ tube.

Cell culture. Pineals, dissected from 3-6-week-old chicks (Cornbelt Hatcheries, Forest, IL) maintained on LD12:12, were rinsed 3 times in a solution of Hanks' salts, 1000 units $/ \mathrm{ml}$ penicillin, $1000 \mu \mathrm{g} / \mathrm{ml}$ streptomycin, $25 \mu \mathrm{g} / \mathrm{ml}$ fungizone and $500 \mu \mathrm{g} / \mathrm{ml}$ gentamicin. The tissue was minced in $2 \mathrm{ml}$ (per 10 glands) of $1 \mathrm{mg} / \mathrm{ml}$ collagenase (132 units $/ \mathrm{mg}$; Cooper Biomedical; Malvern, PA) dissolved in Hanks' salts. Incubation took place in a $37^{\circ} \mathrm{C}$ shaking water bath for $30 \mathrm{~min}$. The partially dispersed fragments wcre mechanically triturated with a fire-polished pasteur pipette, followed by centrifugation for $2 \mathrm{~min}$ at $100 \times \mathrm{g}$. Medium 199 with Hanks' salts, supplemented with 10 mM HEPES buffer, $5 \%$ fetal calf serum (Hazelton, Denver, PA), 10\% horse serum (Gibco, Grand Island, NY), $0.9 \mathrm{mg} / \mathrm{ml} \mathrm{NaHCO}, 100 \mathrm{U} / \mathrm{ml}$ penicillin and $100 \mu \mathrm{g} / \mathrm{ml}$ streptomycin (complete medium) was added to the supernatant, followed by an additional $5 \mathrm{~min}$ spin at $500 \times g$ to harvest dissociated cells. The cell pellet was resuspended in complete medium following centrifugation. Concurrently, papain (Cooper Biomedical), at a concentration of $7 \mathrm{U} / \mathrm{ml}$, in a $4 \mathrm{ml}$ (per 20 glands) solution of Hanks' salts without $\mathrm{Ca}^{2+}$ or $\mathrm{Mg}^{2+}$, was added to the undigested fragments from the original $100 \times g$ pellet. The resulting suspension was incubated for 15 min in a $37^{\circ} \mathrm{C}$ shaking water bath, followed by mechanical trituration with a fire-polished pasteur pipette. Remaining fragments were allowed to settle for approximately $3 \mathrm{~min}$. Complete medium was added to the suspended cells in the papain solution. These cells were collected by centrifugation at $500 \times g$ for $5 \mathrm{~min}$ and resuspended in complete medium. The fragments of undigested tissue that remained following papain digestion were incubated for an additional $15 \mathrm{~min}$ in the same concentration of collagenase and under the same conditions used in the initial digestion. Cells dissociated by this process were collected in the same manner as above. Tissue that remained undigested was discarded. The cells harvested from the 3 separate digestions were then pooled and counted with a hemacytometer. Optimal yield using this procedure was about $1 \times 10^{6} \mathrm{cells} /$ pineal gland.

Falcon (Becton-Dickinson Labware, Oxnard, CA) or Costar (Cambridge, MA) 96-well tissue culture plates were coated with type I calf skin collagen (Calbiochem, La Jolla, CA) in order to facilitate cell attachment. Collagen was treated as sterile and dissolved in a 1:1000 glacial acetic acid solution by stirring overnight at room temperature, followed by storage at $4^{\circ} \mathrm{C}$. Fifty microliters of collagen solution was added to and removed from each well. Plates were air-dried in the tissue culture hood. Cells were plated at a concentration of $2.5 \times 10^{4}$ cells/ well with $125 \mu \mathrm{l}$ of complete medium. On the second day an additional $100 \mu \mathrm{l}$ of complete medium was added to each well (Fig. 1). Because the pineal cell cultures contain photoreceptors and circadian oscillators that regulate melatonin production, the cultures were exposed to a lightdark cycle in vitro in order to synchronize the circadian oscillation of melatonin release (Robertson and Takahashi, 1987). Cultures were maintained on the same I.D12:12 cycle of the chickens from which the pineal glands had been collected. Just prior to the onset of darkness on the fourth $\mathrm{d}$, medium was removed from the cultures by aspiration and $200 \mu \mathrm{l}$ of fresh medium added in order to wash the cells. After $10 \mathrm{~min}$ this medium was replaced with $200 \mu \mathrm{l}$ of fresh medium. Culture medium 


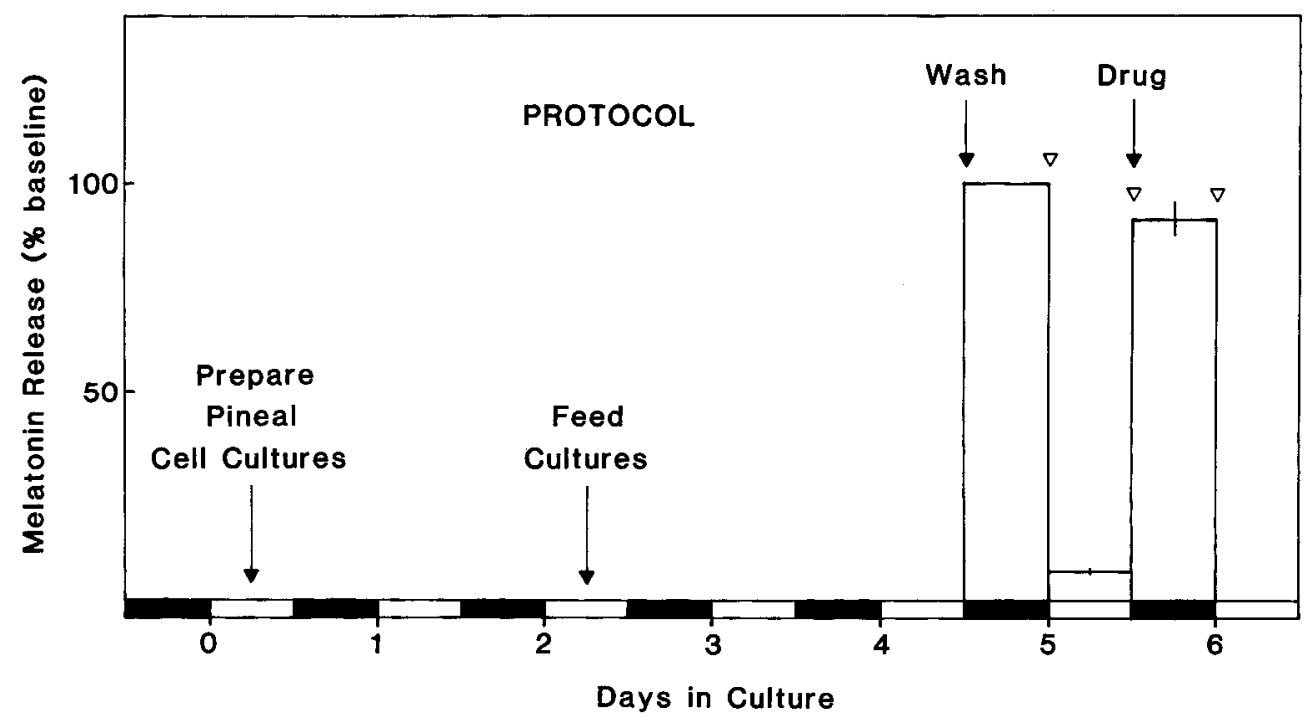

Figure 1. Experimental protocol followed in all experiments. Pineal glands were collected from 3-6-week-old chicks and pineal cell cultures prepared. Cultures were maintained on a $12 \mathrm{hr}$ light: dark cycle, indicated at the bottom. Cultures were fed on day 2 . Just prior to lights off on day 4 , culture medium was changed twice in order to "wash" away the melatonin that had been released into the medium on preceding days. Open triangles indicate when the medium was changed and samples collected. Experimental drugs were administered just prior to lights off on day 5 of culture. The histogram data indicate the mean amount of melatonin released into culture medium over various 12 hr periods by 8 replicate control cultures. The SEM is shown for the $12 \mathrm{hr}$ daytime period and the $12 \mathrm{hr}$ experimental night period. The amount of melatonin released on the baseline night is, by definition, $100 \%$, which corresponds to an average value of $5.0 \mathrm{ng} / 12 \mathrm{hr} /$ well. On the experimental night, these cultures released $91 \%$ of the melatonin that they had released on the preceding night $\pm 4 \%$ SEM. Daytime values were $9 \%$ of the previous night values $\pm 0.004 \%$ SEM.

was then collected every $12 \mathrm{hr}$ in order to obtain "night" and "day" baseline levels of melatonin release, as measured by radioimmunoassay of the medium samples. The cultures were then used in the experiments described below.

Agonist dose-response curves. Immediately after collection of baseline medium samples from which day and night levels of melatonin could be measured, norepinephrine or its agonists were administered in culture medium containing $0.01 \mathrm{~mm}$ sodium ascorbate. This was just prior to the onset of darkness on the fifth d of culture (Fig. 1). Drugs were given in half-log-dose units spanning a range of approximately $10^{-10}-10^{-4} \mathrm{M}$, a range that varied slightly according to the potency of the particular agonist. Each drug dose was given to 8 replicate cultures (one column of the 96-well culture plate). One tissue culture plate was sufficient for each dose curve, with the appropriate control conditions being tested on the same plate as the experimental drug doses. Cell cultures were exposed to norepinephrine or its agonists for the $12 \mathrm{hr}$ night period. Immediately following the onset of light the next day, medium samples were collected to determine the effect of these agonists upon melatonin release. The experiment was then teminated.

Antagonist dose-response curves. The potencies of antagonists were determined by Schild analysis, in which norepinephrine dose-response curves were measured in the presence of various concentrations of each antagonist. Appropriate antagonist concentrations were determined by doing pilot studies in which an antagonist dose-response curve was run in the presence of a $10^{-7} \mathrm{M}$ concentration of norepinephrine. Five-logdose unit norepinephrine concentrations were used in the Schild analysis studies, with the norepinephrine dose range determined by the potency of the particular antagonist concentration. There were 4 replicates per experimental condition and 8 replicates per control condition. Drugs were administered in culture medium containing $0.01 \mathrm{~mm}$ sodium ascorbate just prior to the onset of darkness on the fifth $\mathrm{d}$ of the experiment. The experimental paradigm, in terms of cell culture maintenance and medium sample collections, was the same as that described for agonist studies (Fig. 1).

Analysis. All data are expressed as percentages of melatonin released on the experimental night, as compared to the amount of melatonin released on the baseline night prior to experimental drug administration. Cultures exposed to only sodium ascorbate constituted the control condition to which all agonist and antagonist data were normalized. Normalized data were analyzed by the PREFIT and ALLFIT programs, written by Drs. DeLean, Munson, and Rodbard (DeLean et al., 1978). Nor- malized data for each replicate were entered into the PREFIT program, which yielded a weighting function used in obtaining the weighted statistical fit of the data in ALLFIT. The curve-fitting procedure in ALLFIT is based on the 4-parameter logistic equation $y=\left[(a-d) / 1+\left(X^{\prime} / c^{\prime}\right)^{\prime}\right]+$ $d$, where $a=$ minimum response, $b=$ slope, $c=$ the dose of the drug effective in producing $50 \%$ of the response $\left(\mathrm{EC}_{50}\right), d=$ maximum response, and $X=$ agonist concentration. An $F$ test, defined as the meansquare ratio of a free parameter fit to a fit with various parameter conditions, was calculated. A free parameter fit was done for each group of dose-response curves, followed by a fit in which the $b$ parameter was shared by the curves and a fit in which both the $b$ and $d$ parameters were shared by the curves. FC $_{-50}$ values derived from whichever of the latter 2 fits yielded the smallest $F$ value were used in subsequent analyses. Potency ratios for adrenergic agonists were calculated with reference to the $\mathrm{EC}_{50}$ of $(-)$-norepinephrine.

Schild analysis was used to determine $\mathrm{pA}_{2}$ values for antagonist potencies. Norepinephrine dose-response curves, in the presence of various concentrations of a single antagonist, were analyzed as a group using ALLFIT. The analysis was performed as described above, with a free parameter fit being compared with fits sharing the $b$ parameter or the $b$ and $d$ parameters. Dose ratios obtained from the $\mathrm{EC}_{50}$ values for the norepinephrine dose-response curves measured with different antagonist concentrations were used in the Schild plot. $\mathrm{pA}_{2}$ is defined as that concentration of an antagonist required to shift the effect of a 2-fold dose of norepinephrine to that of a single dose (Schild, 1947). It is obtained by plotting $\log ($ dose ratios -1 ) relative to $-\log$ (each antagonist concentration), where dose ratio is defined as the ratio of the $\mathrm{EC}_{50}$ of each antagonist concentration in the presence of agonist to the $\mathrm{EC}_{50}$ of agonist alone. The $X$-intercept of the regression line for this plot is the $\mathrm{pA}_{2}$ value (Arunlakshana and Schild, 1959). In the case where only one dose of an antagonist competitively shifted the norepinephrine doseresponse curve more than 2 -fold to the right, the $\mathrm{pA}_{2}$ value was derived from the following formula: $\mathrm{pA}_{2}=\log (\operatorname{dose}$ ratio -1$)-\log$ (antagonist concentration), as described in Starke et al., 1983.

Experimental variance. Each of the 22 drugs used was tested on a separate 96-well tissue culture plate, with the exception of (-)-propranolol, SCH23390, and S-sulpiride, which were tested on the same plate. A separate sodium ascorbate control group of 8 replicates was run on each tissue culture plate. Since the $(-)$-norepinephrine curve was run 4 times, we have data on 23 control groups. On the experimental night, these control groups released an average of $82 \pm 2 \%$ SEM of the 


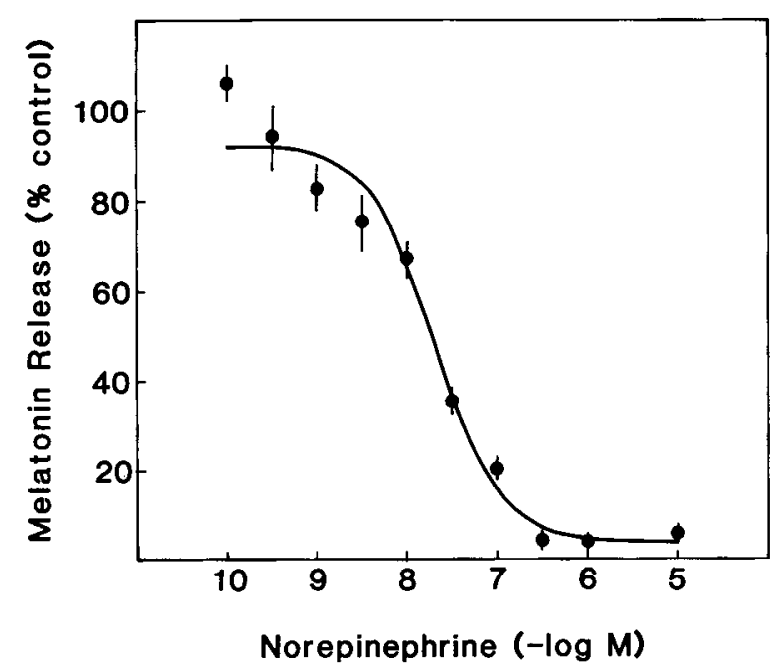

Figure 2. Dose-response curve for (-)-norepinephrine in relation to normalized melatonin release values. Closed circles indicate the normalized mean amount of melatonin released into culture medium over a $12 \mathrm{hr}$ night period by 8 replicate cultures/dose \pm SEM. The curve is a weighted statistical fit of the data using ALLFIT (see text). All data are expressed as the percentage of melatonin released on the experimental night relative to the amount of melatonin released on the baseline night prior to experimental drug administration. These percentage values for experimental cultures were then normalized to the average percentage value of the control group.

melatonin that they had released on the preceding night. The coefficient of variation on interdrug control variability was $11 \%$. The average coefficient of variation of intradrug control variability was $12 \pm 1 \%$ SEM.

The variability in the amount of melatonin released by pineal cell cultures exposed to experimental drugs varied as a function of whether the cultures were exposed to high or low doses. In regard to the 12 adrenergic agonists tested, the average SEM on the lowest dose tested for each was $4.27 \pm 0.43$ SEM. The average SEM on the highest dose tested for each was $0.77 \pm 0.25$ SEM. The unequal distribution of variability in the dose-response curve data is taken into account in the PREFIT program by the use of a weighting function that is calculated when individual replicate data are entered. The parameters of this weighting function are then used to obtain a weighted statistical fit in ALLFIT.
The amount of melatonin released by control cultures during the 12 hr daytime period was measured. These cultures showed a daily rhythm of melatonin release, with release being highest during the $12 \mathrm{hr}$ night period (data cited above and Fig. 1). During the $12 \mathrm{hr}$ daytime period, these control cultures released an average of $9 \%$ of the melatonin that was released on the preceding night $\pm 0.004 \%$ SEM. This daily rhythm of melatonin release is similar to that which has been reported on many occasions in both cell and organ cultures of the avian pineal (Takahashi et al., 1980; Robertson and Takahashi, 1987).

\section{Results}

\section{Effects of adrenergic agonists on melatonin release}

Norepinephrine inhibited melatonin release in a dose-dependent manner, with an average $\mathrm{EC}_{50}$ of $19.7 \mathrm{nM} \pm 2.23 \mathrm{SEM}$ in 4 separate experiments. Norepinephrine is especially effective in avian pineal cell cultures, inhibiting melatonin release to values as low as $4 \%$ of that seen in control cultures (Fig. 2). The dose-response curve for the effect of norepinephrine was monophasic, as were the dose-response curves for all adrenergic agonists tested.

Figure 3 shows that the physiological response to norepinephrine, epinephrine, and isoproterenol is stereospecific, which is to be expected if the physiological response is a receptormediated event. In all 3 cases, the $(-)$ stereoisomer of each catecholamine was more potent than the $(+)$ stereoisomer. Second, the data in Figure 3 show that epinephrine is more potent than norepinephrine, both of which are considerably more potent than isoproterenol (see Table 1 for $\mathrm{EC}_{50}$ values). This relative order of potency is what one would expect of an alphaadrenergic receptor (Langer, 1981; Starke, 1981; Bylund and U'Pritchard, 1983).

To determine the alpha-adrenergic subtype, the effects of alpha- 1 and alpha- 2 adrenergic agonists were tested. The data in Figure 4 suggest that the adrenergic receptor in the avian pineal gland is of the alpha- 2 subtype. Guanabenz acetate and alphamethyl-(-)-norepinephrine, for which dose-response curves are shown in Figure $4 A$, have virtually the same potency as the imidazoline derivatives, oxymetazoline, tramazoline, and clonidine, shown in Figure $4 B$ (see Table 1 for $\mathrm{EC}_{50}$ values). These 5 drugs are more potent than $(-)$-norepinephrine, which is
Figure 3. Dose-response curves for stereoisomers of norepinephrine, epinephrine, and isoproterenol. Points shown indicate the normalized mean amount of melatonin released into culture medium over a $12 \mathrm{hr}$ period by 8 replicate cultures/dose, as indicated by the key (lower left). The curves are weighted statistical fits of the data using ALLFIT (see text), where both the slope and maximal response are the same for all 6 curves $(F=0.61 ; p=0.80)$. The ( ) isomers of norepinephrine, epinephrine, and isoproterenol are depicted as solid lines, and the $(+)$ isomers of each catecholamine are depicted by dashed lines.

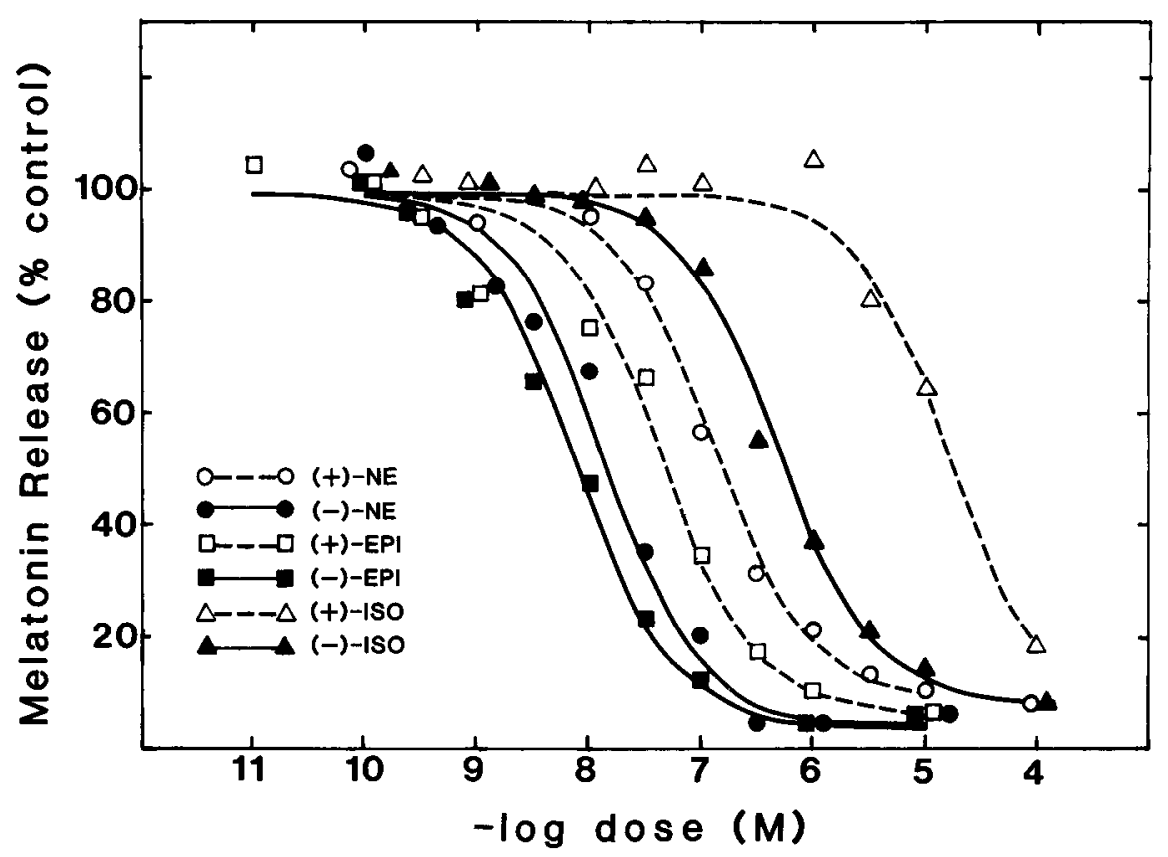




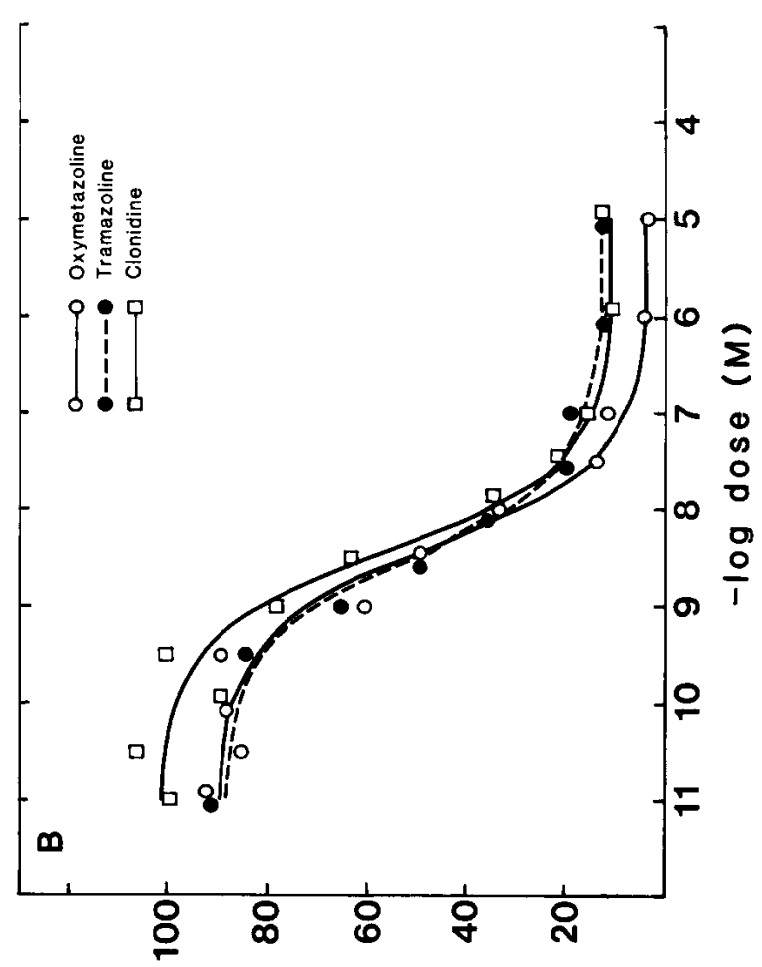

总兽玄号

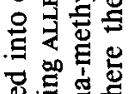

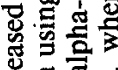

专焉

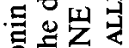

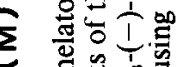

更牙

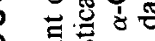

-

음

G.op

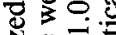

疍 $\|$

药政

욤ㅁㅇㅇ

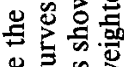

Q

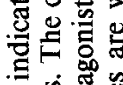

窎兽

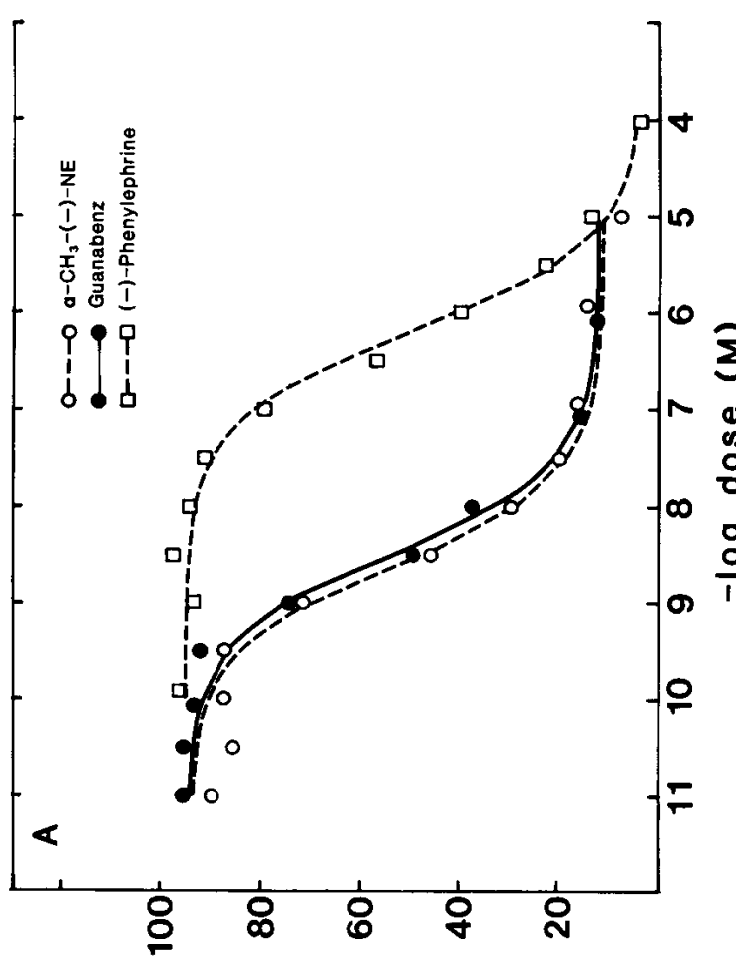

嗐

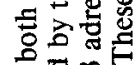

.

熺

की

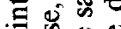

웜․․․

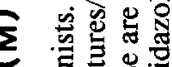

总豆 岂.

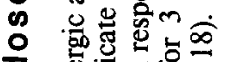

-

요 5 s

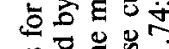

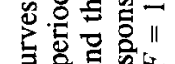

诘语

훙영

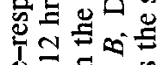

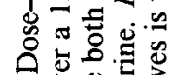

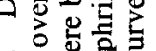

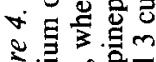

(1017uos \%) eseejey uinojejew 
Table 1. Relative potencies of adrenergic agonists

\begin{tabular}{lcc} 
Drug & $\mathrm{EC}_{50}(\mathrm{nM})^{a}$ & $\begin{array}{l}\text { Potency } \\
\text { ratio }^{b}\end{array}$ \\
\hline Alpha-methyl-(-)-norepinephrine & 2.46 & 0.125 \\
Tramazoline & 3.06 & 0.155 \\
Guanabenz & 3.31 & 0.168 \\
Clonidine & 3.70 & 0.188 \\
Oxymetazoline & 4.29 & 0.218 \\
(-)-Epinephrine & 7.44 & 0.378 \\
(-)-Norepinephrine & 19.7 & 1.00 \\
Dopamine & 37.6 & 1.91 \\
(+)-Epinephrine & 42.6 & 2.16 \\
(+)-Norepinephrine & 125. & 6.35 \\
(-)-Isoproterenol & 463. & 23.5 \\
(-)-Phenylephrine & 659. & 33.5 \\
(+)-Isoproterenol & $17,200$. & 873.
\end{tabular}

${ }^{a}$ Dose-response curves were fitted to the 4-parameter logistic equation, $y=((a-$ d) $\left./ 1+(X / c)^{h}\right)+d$, as described in DeLean et al. (1978). $a=$ Minimum response, $b=$ slope, $c=\mathrm{EC}_{50}, d=$ maximum response, $X=$ agonist concentration.

${ }^{n}$ Potency ratio $=$ ratio of the $\mathrm{EC}_{50}$ of $(-)$-norepinephrine to that of each agonist.

what one would expect of an alpha- 2 receptor. This would not be true of an alpha-1 receptor. In addition, (-)-phenylephrine, a potent alpha-1 agonist, for which the dose-response curve is shown in Figure $4 A$, is not very potent in pineal cell cultures. The $\mathrm{EC}_{50}$ for (-)-phenylephrine, derived from the dose-response curve shown in Figure $4 A$, is 659 nм.

\section{Effects of adrenergic antagonists}

In order to ascertain definitively what type of adrenergic receptor is responsible for mediating the physiological effect of norepinephrine, we determined the relative potency of adrenergic antagonists, as well as of agonists. Figures 5 and 6 show norepinephrine dose-response curves measured in the presence of various concentrations of rauwolscine, yohimbine, phentol- amine, and RX781094. The Schild plots derived from these 4 sets of dose-response curves are shown in the upper-right-hand insets in each of the figures. In all cases, as the concentration of antagonist increases, the apparent potency of norepinephrine decreases. There is no change in the slope of the curves nor in the range of responsiveness, which suggests that the antagonism of these agents is competitive. Using ALLFIT to analyze the data, we found that the fit in which both the slope and the maximum response were shared by the family of dose-response curves when measured in the presence of various concentrations of each antagonist was not significant compared to a free parameter fit. The curves for yohimbine, RX781094, and phentolamine are shown with only the slope as a shared parameter, because that fit yielded a slightly lower $F$ value.

Table 2 lists the $\mathrm{pA}_{2}$ values, derived from Schild analysis, for all adrenergic antagonists tested. As can be seen, rauwolscine, with a $\mathrm{pA}_{2}$ of 9.55 , was the most potent adrenergic antagonist (Fig. 5, top), followed by RX781094 (Fig. 6, bottom). Both of these drugs are known to be very specific for alpha-2 receptors (Perry and U'Pritchard, 1981; Doxey et al., 1983; Hannah et al., 1983) and, indeed, they are very potent in antagonizing norepinephrine's action in avian pineal cell cultures. Yohimbine, another alpha-2 antagonist, has a $\mathrm{pA}_{2}$ value of 8.14 , which is consistent with the presence of alpha- 2 receptors in the avian pineal gland. Prazosin, a specific alpha- 1 adrenergic antagonist, is not very potent, with a $\mathrm{pA}_{2}$ value of 5.93. In addition, there was no shift in the norepinephrine dose-response curve measured in the presence of a $1 \times 10^{-6} \mathrm{M}$ concentration of the betaadrenergic receptor antagonist (-)-propranolol, the D-2 dopamine antagonist $\mathrm{S}$-sulpiride, or the D-1 dopamine antagonist, $\mathrm{SCH} 23390$. Because these specific beta-adrenergic receptor and dopamine receptor antagonists (Creese et al., 1983) were ineffective, these data further support the presence of alpha-2 receptors in avian pineal cell cultures.

The $\mathrm{pA}_{2}$ value for prazosin was derived from a single $1 \times$ $10^{-6} \mathrm{M}$ concentration of prazosin because a $1 \times 10^{-7} \mathrm{M}$ concentration did not shift the norepinephrine dose-response curve

Table 2. $\mathbf{p A}_{2}$ values for antagonists derived from Schild analysis

\begin{tabular}{llclll} 
Drug & $\begin{array}{l}\text { No. } \\
\text { doses }\end{array}$ & $\mathrm{pA}_{2}{ }^{a}$ & $r^{b}$ & Parameter conditions & $F^{c}$ \\
\hline Rauwolscine & 4 & 9.55 & 0.997 & $b$ and $d$ shared & $1.67(p=0.26)$ \\
RX781094 & 3 & 8.32 & 0.999 & $b$ shared & $1.25(p=0.37)$ \\
Yohimbine & 4 & 8.14 & 0.996 & $b$ shared & $1.55(p=0.29)$ \\
Phentolamine & 3 & 7.11 & 0.982 & $b$ shared & $0.15(p-0.93)$ \\
WB4101 & 2 & - & - & $b$ shared $/ d$ constrained & $0.87(p=0.57)$ \\
Prazosin & 2 & $5.93^{r}$ & - & $b$ and $d$ shared & $0.57(p=0.70)$ \\
(-)-Propranolol & 1 & $<6.00^{g}$ & - & $b$ shared & $2.26(p=0.27)$ \\
SCH23390 & 1 & $<6.00^{g}$ & - & $b$ shared & $0.49(p=0.56)$ \\
S-sulpiride & 1 & $<6.00^{g}$ & - & $b$ and $d$ shared & $2.34(p=0.30)$
\end{tabular}

" $\mathrm{pA}_{2}=X$ intercept of the regression line for the Schild plot except where noted. Schild plot $=\log ($ dose ratios -1$)$ relative to $-\log \left(\right.$ antagonist concentrations [AN]). Dose ratio $=$ ratio of $E_{50}$ of each [AN] in the presence of agonist to the $\mathrm{EC}_{50}$ of agonist alone.

$r r=$ Correlation coefficient of the regression line described in $a$.

" Dose-response curves for each of the doses tested for each drug were fitted as a group to the 4-parameter logistic equation, as described in Table 1 .

${ }^{d} F=$ Mean-square ratio of a free parameter fit to a fit with the listed parameter conditions. $p=$ probability level.

Values constrained to those seen in the absence of agonist.

${ }^{2} \mathrm{~A}_{2}$ Value derived from the following formula: $\mathrm{pA}_{2}=\log (\operatorname{dose}$ ratio -1$)-\log [\mathrm{AN}]$, as described in Starke et al (1983).

s No shift in the agonist curve occurred at $1 \times 10^{-6} \mathrm{M}$ [AN]. 


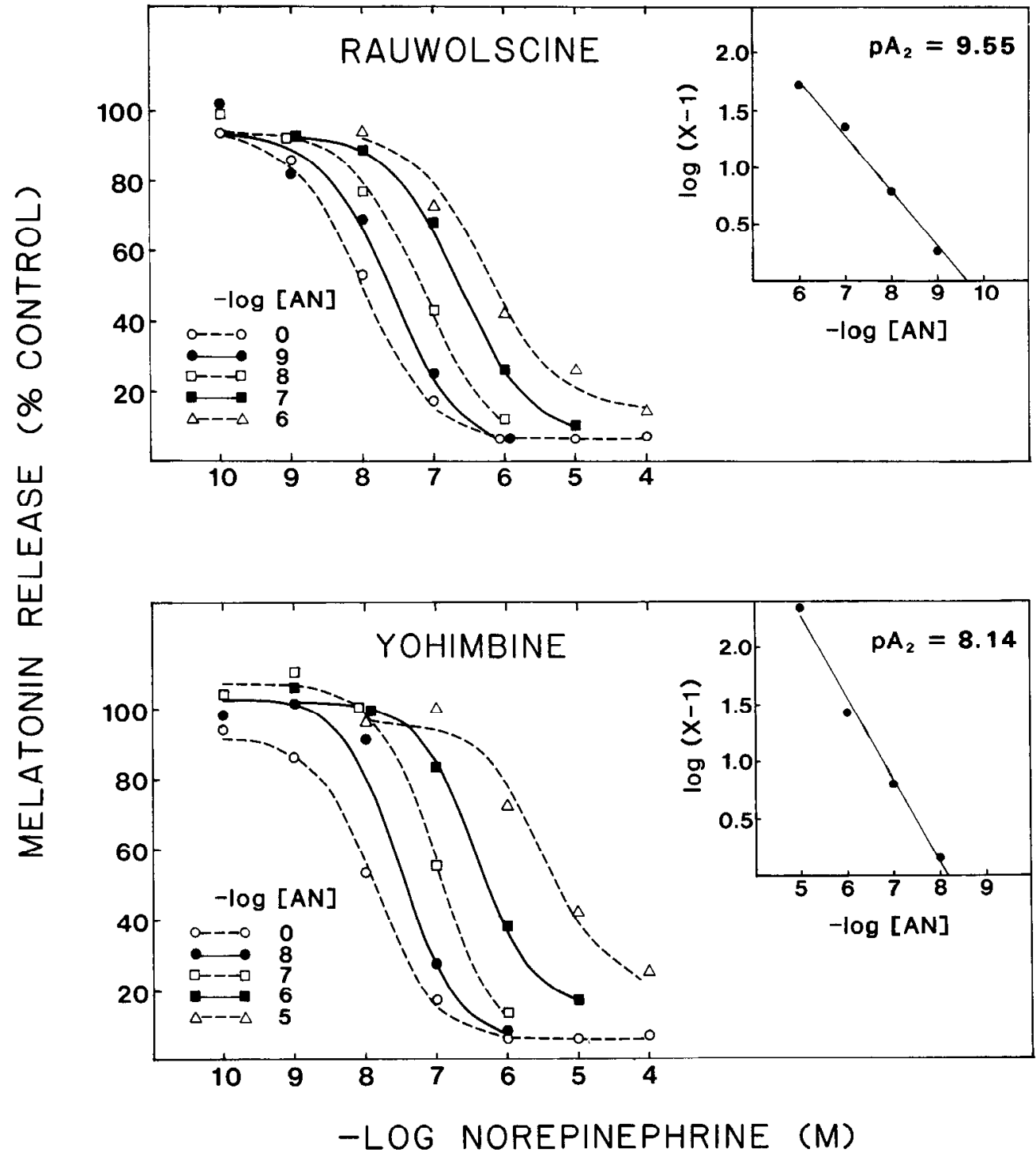

Figure 5. Schild analysis of the adrenergic antagonists, rauwolscine and yohimbine. The lefi-hand sections, upper and lower panels, show norepinephrine dose-response curves measured in the presence of various concentrations of each antagonist. Points shown indicate the normalized mean amount of melatonin released into culture medium over a $12 \mathrm{hr}$ night period by 4 replicate cultures/dose, as indicated by the keys. [AN], molar antagonist concentration. In the case of rauwolscine, the curves are weighted statistical fits of the data, where both the slope and the maximal response are the same for all curves $(F=1.67 ; p=$ 0.26 ). In the case of yohimbine, the curves are weighted statistical fits of the data, where the slope is the same for all curves $(F=1.55 ; p=0.29)$. Inserts both show the Schild plot derived from the dose-response curves on the left. $X=$ The ratio of the $\mathrm{EC}_{50}$ of each antagonist concentration in the presence of agonist to the $\mathrm{EC}_{50}$ of agonist alone. $p A_{2}=X$ intercept of the regression line through the plotted points. 2-fold and a $1 \times 10^{-5} \mathrm{M}$ concentration shifted the dose-response curve in a noncompetitive manner. The norepinephrine doseresponse curve measured in the presence of a $1 \times 10^{-5} \mathrm{M}$ concentration of prazosin was shifted upward, which yielded a maximum response of $130 \%$ at a $1 \times 10^{-9} \mathrm{M}$ concentration of norepinephrine. The curve was very steep, with a slope of -1.74 , an $\mathrm{EC}_{50}$ of $109 \mathrm{nM}$, and a maximum inhibition of $34 \%$ of control at a $1 \times 10^{-5} \mathrm{M}$ norepinephrine concentration. The parameters of the dose-response curves measured in the presence of $1 \times$ $10^{-6}$ and $1 \times 10^{-7} \mathrm{M}$ concentrations were in the normal range, with a slope of -1.03 . A dose-response curve for prazosin, measured in the absence of norepinephrine, showed that pra7osin did not have any agonist effects upon melatonin release.

There is no $\mathrm{pA}_{2}$ value listed for WB4101 because WB4101 appeared to act as a partial agonist. When the effect of WB4101 on melatonin release was tested, in the absence of norepinephrine, across a dose range of $1 \times 10^{-10}$ to $1 \times 10^{-5} \mathrm{M}$, a doseresponse curve with an $\mathrm{EC}_{50}$ of $286 \mathrm{~nm}$ resulted. The curve was shallow with a slope of -0.57 . Melatonin release was inhibited to a value of $28 \%$ of controls. As a result of the inhibition of melatonin release caused by WB4 101 itself, norepinephrine dose- response curves, measured in the presence of either $1 \times 10^{-6}$ or $1 \times 10^{-5} \mathrm{M}$ concentrations of WB4101, were too shallow to be usable in Schild analysis. A $1 \times 10^{-8} \mathrm{M}$ concentration of WB4101 did not shift the norepinephrine dose-response curve 2-fold. The $1 \times 10^{-7} \mathrm{M}$ concentration of WB4101 did shift the norepinephrine dose-response curve to the right in a competitive manner, but since that same dose of WB4101 itself inhibited melatonin release, it was inappropriate to enter that dose ratio into the $\mathrm{pA}_{2}$ formula, where any inhibition of melatonin release would be evaluated as though it were due solely to competitive antagonism. This would, of course, yield an artificially high $\mathbf{p A}_{2}$ value.

\section{Discussion}

These results strongly suggest that postjunctional alpha-2 receptors mediate the regulation of melatonin release by norepinephrine in the pineal gland of the chick. The relative order of potency of both adrenergic agonists and antagonists is identical to that of other alpha-2 adrenergic systems (Langer, 1981; Starke, 1981; Bylund and U'Pritchard, 1983). In addition, there is a good correlation between our drug potency measures, derived 


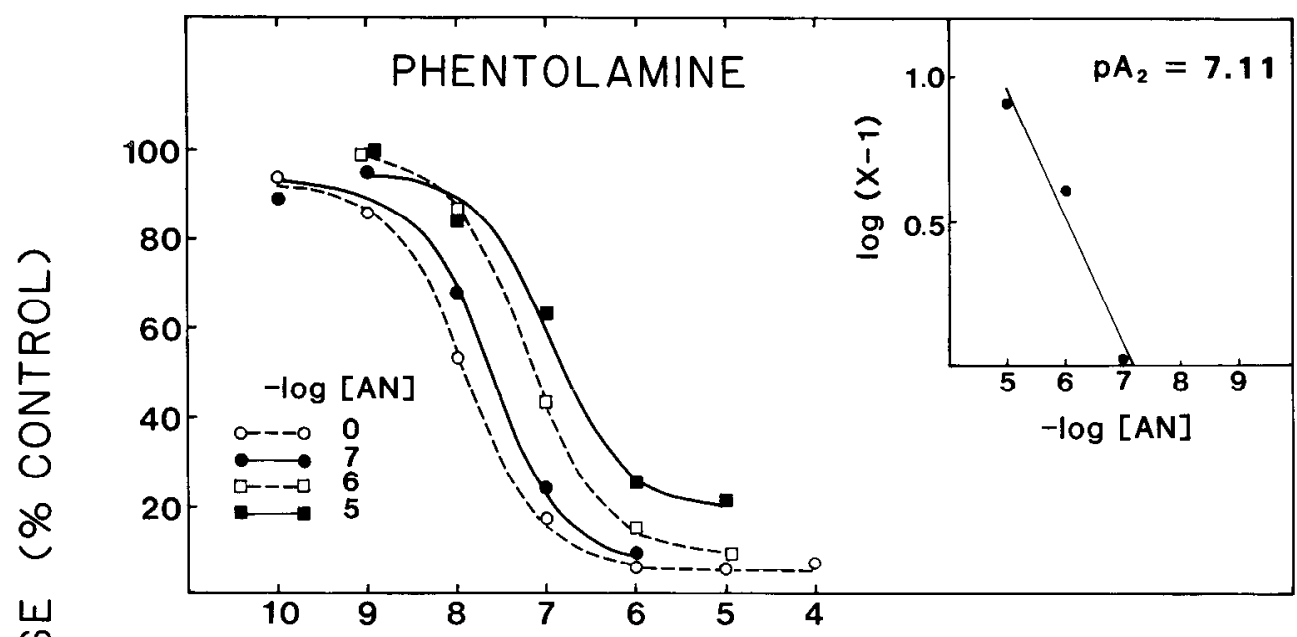

Figure 6. Schild analysis of the adrenergic antagonists phentolamine and RX781094. The dose-response curves (left) and the Schild analysis (right) are similar to those described in Figure 5. In the cases of both phentolamine and RX781094, the curves are weighted statistical fits of the data, where the slope is the same for all curves $(F=0.15 ; p=$ 0.93 and $F=1.25 ; p=0.37$, respectively).

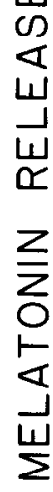

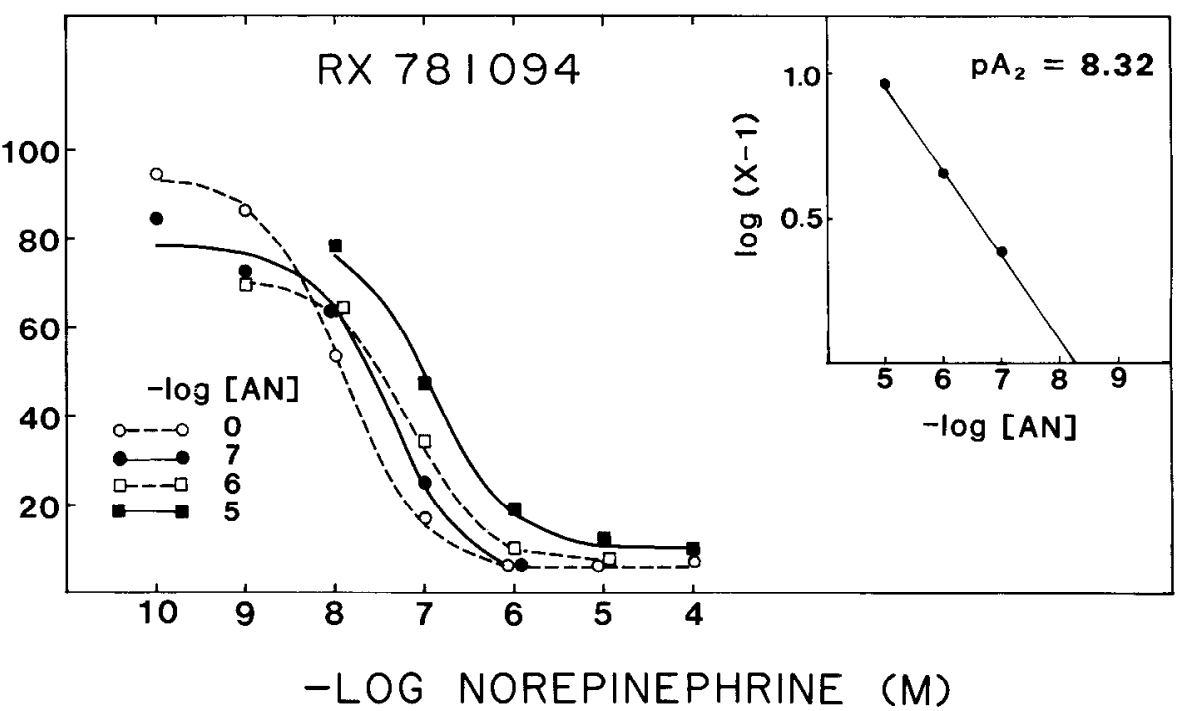

from the physiological studies described above, and the potency measures derived from the same drugs in receptor binding studies that used alpha-2 radioligands. No such correlation was found when the comparison was made with data derived from alpha- 1 radioligands. When we plotted our $\mathrm{EC}_{50}$ values for agonists and $\mathrm{pA}_{2}$ values for antagonists against $K_{\mathrm{i}}$ values obtained in binding studies, we obtained a slope close to 1 and a significant correlation between the two in regard to 4 different alpha- 2 ligands used in neural tissue. In the comparison of our data with those of U'Pritchard and Snyder (1977) using calf frontal cortex, we found a slope of 1.07 and a correlation of 0.83 with regard to ${ }^{3} \mathrm{H}$-norepinephrine and a slope of 1.07 and a correlation of 0.81 for ${ }^{3} \mathrm{H}$-epinephrine ( 11 drugs in common; $n=11$ ). Comparing $K_{\mathrm{i}}$ values obtained in rat brain minus cerebellum to our data, using ${ }^{3} \mathrm{H}$-clonidine as the ligand (U'Pritchard et al., 1977), yielded a slope of 1.02 and a correlation of $0.90(n=15)$. Identification of adrenergic binding sites through the use of ${ }^{3} \mathrm{H}$-paraaminoclonidine in rat cerebral cortex (Rouot and Snyder, 1979) yielded $K_{\mathrm{i}}$ values with a correlation of 0.81 and a slope of 0.81 $(n=9)$. The only drug whose potency consistently varied between receptor binding studies and physiological studies was phentolamine. Phentolamine, known to be a general alpha an- tagonist, had a lower potency in chick pineal cell cultures, which is in accord with previously reported physiological studies (Wikberg, 1979). This potency is less than that which is generally found in receptor binding studies.

Receptor subtype identification gives us an idea of the most probable biochemical series of events initiated by receptor activation. It is well known that beta-adrenergic receptor activation stimulates adenylate cyclase, which, in turn, increases cAMP. Alternatively, alpha-adrenergic receptor activation is associated either with phospholipid turnover and calcium mobilization changes, in the case of alpha-1 receptors, or cAMP inhibition in the case of alpha-2 receptors (Limbird, 1983; Sugden et al., 1985a). In the rat, it is well known that beta-adrenergic receptors and adenylate cyclasc, acting via their stimulatory effects on cAMP, are involved in NAT induction (Zatz et al., 1978). Qualitative data suggest that cAMP is also involved in regulating NAT activity in the pineal gland of the chick. In organ-cultured pineals, an increase in NAT activity was seen in response to phosphodiesterase inhibitors, cAMP analogs, and cholera toxin (Deguchi, 1979b).

We have begun to test whether an inhibition of cAMP, caused by norepinephrine-induced alpha- 2 receptor activation, is in- 
volved in the regulation of melatonin release in avian pineal cell cultures. First, if a reduction in cAMP is mediating the norepinephrine signal, then preventing a reduction in CAMP should block the response to norepinephrine. Increasing cAMP levels by introducing cAMP analogs, activating adenylate cyclase with forskolin, or inhibiting phosphodiesterase with IBMX blocks the inhibition of melatonin release in a dose-dependent manner (unpublished results). Second, it is known that in most systems, alpha-2 activation appears to work through an inhibition of adenylate cyclase (Limbird, 1981). This inhibition is mediated by a guanine nucleotide regulatory protein known as $N_{i}$ or $G_{i}, G_{i}$ can be inactivated by pertussis toxin. If norepinephrine decreases cAMP by inhibiting adenylate cyclase, then toxin pretreatment should block the inhibition of melatonin release caused by norepinephrine. In preliminary experiments, pertussis toxin completely blocked the effects of norepinephrine (unpublished results). Since pertussis toxin inactivates $G_{i}$, these data suggest that the inhibition of cAMP levels, which may occur as a result of norepinephrine stimulation, may occur at the level of adenylate cyclase.

In both the rat and chick pineal, an increase of cAMP appears to lead to an increase in NAT, which, in turn, leads to an increase in melatonin synthesis. Therefore, it is clear that the alteration in the physiological effect of norepinephrine from a stimulatory one in the rat to an inhibitory one in the chick occurs at some level upstream from cyclic nucleotide regulation. Having identified alpha-2 adrenergic receptors in the pineal gland of the chick, it appears that this signal conversion occurs at the level of the receptor, since beta receptors lead to increased cAMP levels and alpha-2 receptors lead to decreased cAMP levels.

In this context, it should be remembered that the daily rhythm of melatonin release and/or synthesis is the same in both the chick and the rat. Melatonin levels are high at night and low during the day. The prediction that the daily rhythm of norepinephrine is $180^{\circ}$ out of phase between the 2 species has been experimentally confirmed (Cassone et al., 1986). In chicks, norepinephrine, which is inhibitory to melatonin synthesis, has high turnover rates in the daytime, whereas in rats, norepinephrine, which is stimulatory to melatonin synthesis, has high turnover rates at night (Brownstein and Axelrod, 1974). In summary, it is clear that the conservation of a nocturnal rise of melatonin across a wide range of vertebrates does not imply a common mechanism of action.

There is evidence suggestive that norepinephrine may influence the circadian regulation of melatonin rhythmicity in chicks. First, a circadian rhythm of melatonin release is maintained for as long as 2 weeks when intact chickens are maintained in constant darkness (Ralph et al., 1974), whereas, rhythms of melatonin release from in vitro pineal glands cultured in constant darkness damp after approximately 4 cycles. The possibility that neural input to the gland is responsible for the maintenance of pineal rhythmicity is suggested by the fact that if intact chickens are ganglionectomized, melatonin rhythmicity damps out after 2-3 cycles in constant darkness, just as it does in the in vitro preparation (Cassone and Menaker, 1983). Plasma melatonin rhythms are unaffected by ganglionectomy in a light-dark cycle, suggesting that both photic cues and neural sympathetic input can individually maintain pineal rhythmicity when the 2 factors are experimentally separated. Whether neural input to the gland is acting as an entrainer of circadian rhythmicity as well as an acute regulator of melatonin synthesis is unknown.

There is some data to suggest that norepinephrine adminis- tration may shift the phase of the melatonin rhythm (Takahashi, 1981; Cassone and Menaker, 1983). However, conclusive proof that norepinephrine influences the phase of the oscillation awaits further evidence that a steady-state phase shift has occurred. Until this is demonstrated, the possibility remains that the effects of norepincphrinc on the phase of the melatonin rhythm are caused by norepinephrine's established inhibitory role. If norepinephrine additionally acts as an entraining agent, there may be 2 inputs to the circadian oscillators within the pineal: an input from the photoreceptors within the gland and a central, neural input mediated by norepinephrine. This adrenergic input could carry photic information from photoreceptors outside the pineal (either ucular or extraocular), or it could mediate coupling from another circadian oscillator-perhaps the suprachiasmatic nucleus (Takahashi and Menaker, 1982, 1984b). It is interesting to note that both the suprachiasmatic nuclei and the pineal gland have been implicated as components of the circadian system in the bird, so that the study of the neural flow of information between them may also be the study of central circadian organization.

\section{References}

Ariëns Kapper, (1965) Survey of the innervation of the epiphysis cerebi and the accessory pineal organs of vertebrates. Prog. Brain Res. 10: $87-153$.

Arunlakshana, O., and H. O. Schild (1959) Some quantitative uses of antagonists. Br. J. Pharmacol. 14: 48-58.

Auerbach, D. A., D. C. Klein, C. Woodard, and G. D. Auerbach (1981) Neonatal rat pinealocytes: Typical and atypical characteristics of $\left.{ }^{[125} \mathrm{I}\right]$ iodohydroxybenzylpindolol binding and adenosine $3^{\prime}, 5^{\prime}$-monophosphate accumulation. Endocrinology 108: 559-567.

Bagnara, J. T., and M. E. Hadley (1970) Endocrinology of the amphibian pineal. Am. Zool. 10: 201-216.

Besharse, J. C., D. A. Dunis, and M. Iuvone (1984) Regulation and possible role of serotonin $N$-acetyltransferase in the retina. Fed. Proc. 43: 2704-2708.

Binkley, S. A., J. B. Riebman, and K. B. Reilly (1978) The pineal gland: A biological clock in vitro. Science 202: 1198-1201.

Bowers, C. W., and R. E. Zigmond (1982) The influence of the frequency and pattern of sympathetic nerve activity on serotonin $N$-acetyltransferase in the rat pineal gland. J. Physiol. (Lond.) 330: 279296.

Brownstein, M. J., and J. Axelrod (1974) Pineal gland: 24 hour rhythm in norepinephrine turnover. Science 184: 163-165.

Bylund, D. B., and D. C. U'Pritchard (1983) Characterization of alpha-1 and alpha-2 adrenergic receptors. Int. Rev. Neurobiol. 24: 343-431.

Cassone, V. M., and M. Menaker (1983) Sympathetic regulation of chicken pineal rhythms. Brain Res. 272: 311-317.

Cassone, V. M., J. S. Takahashi, C. D. Blaha, R. F. Lane, and M. Menaker (1986) Dynamics of noradrenergic circadian input to the chicken pineal gland. Brain Res. 384: 334-341.

Creese, I., D. R. Sibley, M. W. Hamblin, and S. E. Leff (1983) The classification of dopamine receptors. Annu. Rev. Neurosci. 6: 43-71.

Deguchi, T. (1979a) Circadian oscillator in cultured cells of chicken pineal gland. Nature 282: 94-96.

Deguchi, T. (1979b) Role of adenosine 3',5'-monophosphate in the regulation of circadian oscillation of serotonin $N$-acetyltransferase activity in cultured chicken pineal gland. J. Neurochem. 33: 45-61.

Deguchi, 'T. (1979c) Circadian rhythm of serotonin $N$-acetyltransferase activity in organ culture of chicken pineal gland. Science 203: $1245-1247$.

Deguchi, T. (1981) Rhodopsin-like photosensitivity of isolated chicken pineal gland. Nature 290: 706-707.

DeLean, A., P. J. Munson, and D. Rodbard (1978) Simultaneous analysis of families of sigmoidal curves: Application to bioassay, radioligand assay, and physiological dose-response curves. Am. J. Physiol. 235: E97-E102.

Doxey, J. C., A. G. Roach, and C. F. C. Smith (1983) Studies on RX781094: A selective, potent and specific antagonist of alpha-2adrenoceptors. Br. J. Pharmacol. 78: 489-505.

Goldman, B. D. (1983) The physiology of melatonin in mammals. In 
Pineal Research Reviews, vol. 1, R. J. Reiter, ed., pp. 145-182, Liss, New York.

Gwinner, E., and I. Benzinger (1978) Synchronization of a circadian rhythm in pinealectomized European starlings by daily injections of melatonin J. Comp. Physiol. 127: 209-213.

Hamm, H. E., J. S. Takahashi, and M. Menaker (1983) Light-induced decrease of scrotonin $\mathrm{N}$-acctyltransferasc activity and melatonin in the chicken pineal gland and retina. Brain Res. 266: 287-293.

Hannah, J. A. M., C. A. Hamilton, and J. L. Reid (1983) RX781094, a new potent alpha-2 adrenoceptor antagonist. Naunyn Schmeidebergs Arch. Pharmacol. 322: 221-227.

Kasal, C. A., M. Menaker, and J. R. Perez-Polo (1979) Circadian clock in culture: $N$-acetyltransferase activity of chick pineal glands oscillates in vitro. Science 203: 656-658.

Klein, D. C., and R. Y. Moore (1979) Pineal $N$-acetyltransferase and hydroxyindole- $O$-methyltransferase: Control by the retinohypothalamic tract and the suprachiasmatic nucleus. Brain Res. 174: 245262.

Klein, D. C., D. A. Auerbach, M. A. A. Namboodiri, and G. H. T. Wheler (1981) Indole metabolism in the mammalian pineal gland. In The Pineal Gland: Anatomy and Biochemistry, R. J. Reiter, cd., pp. 199-227, CRC Press, Boca Raton, FL.

Langer, S. Z. (1981) Presynaptic regulation of the release of catecholamines. Pharmacol. Rev. 32: 337-362.

Limbird, L. E. (1983) Alpha-2 adrenergic systems: Models for exploring hormonal inhibition of adenylate cyclase. Trends Pharmacol. Sci. 4: 135-138.

Perry, B. D., and D. C. U'Pritchard (1981) [ $\left.{ }^{3} \mathrm{H}\right]$ Rauwolscine (alphayohimbine): A specific antagonist radioligand for brain alpha-2 adrenergic receptors. Eur. J. Pharmacol. 76: 461-464.

Ralph, C. L., R. W. Pelham, S. E. MacBride, and D. P. Reilly (1974) Persistent rhythms of pineal and serum melatonin in cockerels in continuous darkness. J. Endocrinol. 63: 319-324.

Ralph, C. L., S. Binkley, S. E. MacBride, and D. C. Klein (1975) Regulation of pineal rhythms in chickens: Effects of blinding, constant light, constant dark and superior cervical ganglionectomy. Endocrinology 97: 1373-1378

Robertson, L. M., and J. S. Takahashi (1987) Circadian clock in cell culture. II. In vitro photic entrainment of melatonin oscillation from dissociated chick pineal cells. J. Neurosci. (in press).

Kollag, M. D., and G. D. Niswender (1976) Radioimmunoassay of serum concentration of melatonin in sheep exposed to different lighting regimens. Endocrinology 98: 482-489.

Rouot, B. R., and S. H. Snyder (1979) [ $\left.{ }^{3} \mathrm{H}\right]$ Para-amino-clonidine: A novel ligand which binds with high affinity to alpha-adrenergic receptors. Life Sci. 25: 769-774.

Sato, T., and K. Wake (1983) Innervation of the avian pineal organ. Cell Tissue Res. 233: 237-264.

Schild, H. O. (1947) pA, A new scale for the measurement of drug antagonism. Br. J. Pharmacol. 2: 189-206.

Starke, K. (1981) Alpha adrenoceptor subclassification. Rev. Physiol. Biochem. Pharmacol. 88: 199-236.

Starke, K., L. Spath, J. D. Lang, and C. Adelung (1983) Further func- tional in vitro comparison of pre- and postsynaptic dopamine receptors in the rabbit caudate nucleus. Naunyn Schmeidebergs Arch. Pharmacol. 323: 298-306.

Sugden, D., and D. C. Klein (1984) Rat pineal alpha-1 adrenoceptors: Identification and characterization using [ ${ }^{125}$ I]Iodo-2-[ $\beta$-[4-hydroxyphenyl]-ethylaminomethyl] tetralone. Endocrinology 114:435-440.

Sugden, D., J. L. Weller, D. C. Klein, K. L. Kirk, and C. R. Creveling (1984) Alpha-adrenergic potentiation of beta-adrenergic stimulation of rat pineal $\mathrm{N}$-acetyltransferase. Biochem. Pharmacol. 33: $3947-$ 3950

Sugden, D., J. Vanecek, D. C. Klein, T. P. Thomas, and W. B. Anderson (1985a) Activation of protein kinase $C$ potentiates isoprenaline-induced cyclic AMP accumulation in rat pinealocytes. Nature 314:359361.

Sugden, D., M. A. A. Namboodiri, D. C. Klein, I. E. Pierce, J. Grady, Jr., and I. N. Mefford (1985b) Ovine pineal alpha-1 adrenoceptors: Characterization and evidence for a functional role in the regulation of serum melatonin. Endocrinology 116: 1960-1967.

Takahashi, J. S. (1981) Neural and endocrine regulation of avian circadian systems. Ph.D. dissertation, University of Oregon.

Takahashi, J. S., and M. Menaker (1982) Role of the suprachiasmatic nuclei in the circadian system of the house sparrow, Passer domesticus. J. Neurosci. 2: 815-828.

Takahashi, J. S., and M. Menaker (1984a) Multiple redundant circadian oscillators within the isolated avian pineal gland. J. Comp. Physiol. 154: 435-440.

Takahashi, J. S., and M. Menaker (1984b) Circadian rhythmicity: Regulation in the time domain. In Biological Regulation and Development, vol. 3B, R. F. Goldberger and K. R. Yamamoto, eds., pp. 285-303, Plenum, New York.

Takahashi, J. S., H. Hamm, and M. Menaker (1980) Circadian rhythms of melatonin release from individual superfused chicken pineal glands in vitro. Proc. Natl. Acad. Sci. U.S.A. 77: 2319-2322.

Underwood, H., and M. Harless (1985) Entrainment of the circadian activity rhythm of a lizard to melatonin injections. Physiol. Behav. 35: 267-270.

U'Pritchard, D. C., and S. H. Snyder (1977) Binding of ${ }^{3} \mathrm{H}-$ catecholamines to alpha-noradrenergic receptor sites in calf brain. J. Biol. Chem. 252: 6450-6463.

U'Pritchard, D. C., D. A. Greenberg, and S. H. Snyder (1977) Binding characteristics of a radiolabelled agonist and antagonist at central nervous system alpha noradrenergic receptors. Mol. Pharmacol. 13. 454-473.

Wainwright, S. D., and L. K. Wainwright (1980) Regulation of the cycle in chick pineal serotonin $N$-acetyltransferase activity in vitro by light. J. Neurochem. 35: 451-457.

Wikberg, J. E. S. (1979) The pharmacological classification of adrenergic alpha- 1 and alpha- 2 receptors and their mechanisms of action. Acta Physiol. Scand. (Suppl.) 468: 1-99.

Zatz, M., J. W. Kebabian, and F. O'Dea (1978) Regulation of $\beta$-adrenergic function in the rat pineal gland. In Receptors and Hormone Action, vol. 3, L. Birnbaumer and B. W. O'Malley, eds., pp. 195219, Academic, New York. 